This is the final peer-reviewed accepted manuscript of:

Miglioli, I., et al. "Director Configuration in the Twist-Bend Nematic Phase of CB11CB." Journal of Materials Chemistry C, vol. 4, no. 41, 2016, pp. 9887-9896.

The final published version is available online at : http://dx.doi.org/10.1039/C6TC03246J

Rights / License:

The terms and conditions for the reuse of this version of the manuscript are specified in the publishing policy. For all terms of use and more information see the publisher's website.

This item was downloaded from IRIS Università di Bologna (https://cris.unibo.it/)

When citing, please refer to the published version. 
Cite this: DOI: $10.1039 / x x x x x x x x x x$

Accepted Date

DOI: $10.1039 / x x x x x x x x x x$

www.rsc.org/journalname

\section{Director configuration in the twist-bend nematic phase of CB11CB}

\author{
Isabella Miglioli, ${ }^{a}$ Corrado Bacchiocchi, ${ }^{* b}$ Alberto Arcioni, ${ }^{a}$ Alexandra Kohlmeier, ${ }^{c}$ Georg \\ H. Mehl, ${ }^{c}$ and Claudio Zannoni ${ }^{a}$
}

\begin{abstract}
The director distribution in the nematic phases exhibited by the $1^{\prime \prime}, 11^{\prime \prime}$-bis(4-cyanobiphenyl-4'yl)undecane (CB11CB) liquid crystal has been studied in the bulk with the EPR spin probe technique. EPR spectra confirmed the presence of an higher temperature uniaxial nematic phase and of a lower temperature nematic phase in which the director distribution is not uniform. Spectra recorded in the lower temperature nematic phase were not fully compatible with theoretical EPR spectra calculated according to the recently proposed model for the twist-bend phase in which the local domain director twists around an axis with a fixed tilt angle, $\theta_{0}$, but were well described by a "distributed-tilt" model in which the director has a relatively narrow distribution, centred at $\theta_{0}$.
\end{abstract}

\section{Introduction}

The presence of a second, lower temperature nematic phase in banana-shaped liquid crystals (LCs) made by two cyanobiphenyl mesogenic units linked by an odd-membered alkyl chain (odd symmetric LC dimers) was first observed by Panov et al. ${ }^{1}$. The basic structural features of this phase for the odd symmetric LC dimer homologue $1^{\prime \prime}, 7^{\prime \prime}$-bis(4-cyanobiphenyl-4'-yl)heptane (CB7CB) were then proposed by Cestari et al. ${ }^{2}$ who suggested the phase to be the twist-bend nematic $\left(\mathrm{N}_{\mathrm{tb}}\right)$ phase originally proposed by Meyer ${ }^{3}$ and theoretically predicted by Dozov for banana-shaped mesogens ${ }^{4}$. In particular, Cestari et al. ${ }^{2}$ described the phase as macroscopically uniaxial with a local nematic director that makes a constant tilt angle $\theta_{0}$ with the macroscopic axis and twists around it in an helical fashion. Equal regions of opposite twist are expected to be present making the whole system achiral.

In the following years a number of studies on odd symmetric LC dimers, in particular CB7CB, have supported the twist-bend model for the lower temperature nematic phase. These works include, in particular, a detailed 2D NMR study ${ }^{5}$; a comparison of a molecular field model of the phase to NMR quadrupolar splittings ${ }^{6}$; electro-optic measurements ${ }^{7,8}$; DSC and optical polarization microscopy ${ }^{9}$; freeze-fracture $\mathrm{TEM}^{8,10,11}$ and hi-res cryo$\mathrm{TEM}^{11}$; synchrotron X-ray diffraction ${ }^{10}$ and birefringence mea-

\footnotetext{
a Dipartimento di Chimica Industriale "Toso Montanari”, Università, Viale Risorgimento 4, I-40136 Bologna, Italy

${ }^{* b}$ School of Science and Technology, Chemistry Division, University of Camerino, Via S. Agostino 1, I-62032 Camerino (MC), Italy, Fax: +390737 637 345; Tel: +39 0737 40 2260; E-mail: corrado.bacchiocchi@unicam.it

${ }^{c}$ Department of Chemistry, University of Hull, Hull HU6 7RX, UK
}

surements ${ }^{12}$. A recent work by Tamba et al. ${ }^{13}$ combined optical polarization microscopy, small-angle X-ray scattering and electron microscopy to study the nanostructure and the filament formation properties of a LC phase of a symmetric difluoroterphenyl dimer below the $\mathrm{N}_{\mathrm{tb}}$ phase.

As the twist-bend model for the lower temperature nematic phase gained general consensus, studies emerged trying to elucidate the main features responsible for the onset of the phase and even if the main mechanism responsible for the $\mathrm{N}_{\mathrm{tb}}$ phase is still an open question, some agreement on empirical observations is emerging. In a recent two-part review on the relationship between molecular structure and the formation of the $\mathrm{N}_{\mathrm{tb}}$ phase, Mandle et al. ${ }^{14,15}$ concluded that the overall bent shape of the molecule is more important than electrostatic interactions that nonetheless play a role and must be in a "Goldilocks" range. Similar conclusions have been reached in previous theoretical ${ }^{16}$ and experimental ${ }^{17-19}$ works.

Despite the general agreement, the local director configuration of the $\mathrm{N}_{\mathrm{tb}}$ phase is still debated ${ }^{20,21}$ and a more direct evidence that the director distribution in these materials is exactly of the type indicated by several authors as the heliconical twist-bend is still missing and worth investigating ${ }^{9,22,23}$.

The EPR spin probe technique ${ }^{24-26}$ seems to be well suited to test the proposed structure of the $\mathrm{N}_{\mathrm{tb}}$ phase and, in principle, would be an independent approach to estimate the constant tilt angle $\theta_{0}$. In fact, the analysis of the spectra of a suitable stable nitroxide free radical dissolved in the LC host can provide molecular-level information about the ordering, the reorientational dynamics and the local nematic director configuration sensed by the probe in the $\mathrm{N}_{\mathrm{tb}}$ phase. 
Until now the only EPR measurements on this type of dimers we are aware of have been done by Cestari et al. in their seminal article $^{2}$ on the homologue CB7CB. In a qualitative assessment of the EPR spectra the Authors pointed out that in the $\mathrm{N}$ phase the spectra are consistent with a nematic monodomain whose director is parallel to the magnetic field whereas in the $\mathrm{N}_{\mathrm{tb}}$ phase the spectra change significantly indicating a nonuniform alignment of the director. A detailed analysis of the EPR spectra was however not presented at the time.

Given the proposed structure of the $\mathrm{N}_{\mathrm{tb}}$ phase, this spectral change is not the expected one since, on cooling from a nematic monodomain, it appears reasonable that the axis of the $\mathrm{N}_{\mathrm{tb}}$ phase, around which the director twists with the constant tilt angle $\theta_{0}$, would be parallel to the magnetic field of the EPR spectrometer, as it has been determined in NMR measurements on $\mathrm{CB}^{2} \mathrm{CB}^{27}$. The expected spectrum in the $\mathrm{N}_{\mathrm{tb}}$ phase should therefore correspond still to a monodomain whose director is tilted at an angle $\theta_{0}$ with respect to the magnetic field.

To check if this spectral change is common to other odd symmetric LC dimer homologues, this article presents a detailed investigation of $1^{\prime \prime}, 11^{\prime \prime}$-bis(4-cyanobiphenyl-4'-yl)undecane (CB11CB) in the bulk and provides evidence from the analysis of EPR spectra that seems to be at variance with the proposed description of the $\mathrm{N}_{\mathrm{tb}}$ phase $2,5-8,10-12,27$.

The structure of the article is as follows. In the next section experimental details are provided. The Theory section describes the models used to analyse the EPR spectra. Information recovered from the analyses are then presented in the Results and Discussion section and a summary of the main findings is reported in the Conclusions section.

\section{Experimental}

The LC dimer CB11CB is formed by two cyanobiphenyl groups linked by an alkyl chain of eleven carbon atoms. It was synthesized in the Department of Chemistry at the University of Hull (UK) and was used without further purification. Its molecular structure is shown in Figure 1.



Fig. 1 Molecular structure of $\mathrm{CB} 11 \mathrm{CB}$.

This material, upon cooling from the isotropic (I) phase, exhibits a transition to the ordinary, uniaxial nematic phase $\left(\mathrm{N}_{\mathrm{u}}\right)$ and, on further cooling, to a second nematic phase, described as a $\mathrm{N}_{\mathrm{tb}}$ phase. Phase sequence reported by various authors varies slightly ${ }^{1,9,28-30}$.

The phase sequence of the CB11CB LC used in this work, determined on heating by DSC at $10 \mathrm{~K} / \mathrm{min}$, was: Crystal $-377.2 \mathrm{~K}$ $-\mathrm{N}_{\mathrm{tb}}-381.9 \mathrm{~K}-\mathrm{N}_{\mathrm{u}}-398.5 \mathrm{~K}-\mathrm{I}$.

The nitroxide spin probe, used for doping the CB11CB LC, was the $3 \beta$-doxyl- $5 \alpha$-cholestane free radical (Aldrich, hereafter referred to as CSL). This was employed in a number of previous studies $^{25,31-33}$ where it proved to be a reliable probe to monitor the order and the dynamics of the LC system, due to its size, morphology and rigidity, which results in a strong orientation by the LC host. The CSL structure is shown in Figure 2 together with the chosen ordering ( $\mathrm{x}, \mathrm{y}, \mathrm{z}$, solid line) and magnetic ( $\mathrm{x}^{\prime}, \mathrm{y}^{\prime}, \mathrm{z}^{\prime}$, dashed line) molecular frames and the indication of its two main reorientational motions, tumbling and spinning, with the corresponding components of the rotational diffusion tensor: $D_{\perp}$ (reorientation of the molecular long axis) and $D_{\|}$(rotation around the long axis), respectively.

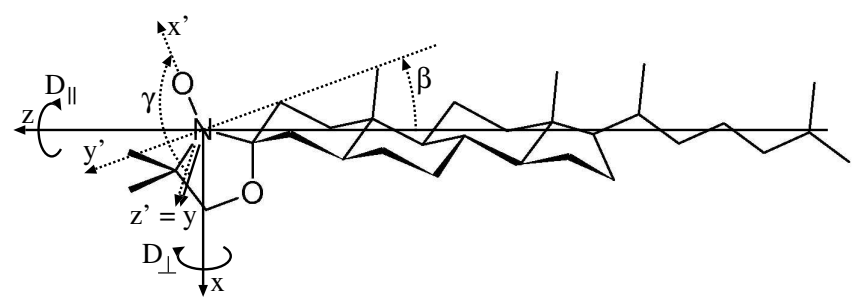

Fig. 2 Molecular structure of the CSL spin probe together with the chosen ordering $\left(x, y, z\right.$, solid line) and magnetic $\left(x^{\prime}, y^{\prime}, z^{\prime}\right.$, dashed line) molecular frames, the Euler angles, $\beta$ and $\gamma$, between the molecular frames and the principal components, $D_{\perp}$ (reorientation of the molecular long axis) and $D_{\|}$(rotation around the molecular long axis), of the rotational diffusion tensor.

CSL was added to CB11CB in the I phase at a typical concentration of about $1 \times 10^{-4} g_{\mathrm{CSL}} / g_{\mathrm{CB} 11 \mathrm{CB}}$, which is below the limiting concentration typically suggested ${ }^{24}$ to avoid Heisenberg spin exchange distortion effects.

Samples of CSL-doped CB11CB were inserted into glass capillaries of $1.8 \mathrm{~mm}$ internal diameter for the EPR measurements. EPR spectra were acquired with a Bruker EMX spectrometer equipped with an ER 041XG microwave X-band (9.5 GHz) Gunn Diode bridge and a rectangular ER 4102ST cavity. The samples were thermostated with a nitrogen flux through a variable temperature unit Bruker B-VT 2000. The temperature was monitored with a calibrated type $\mathrm{T}$ thermocouple (Comark Ltd.) kept in close proximity with the sample and showed a stability better than $\pm 0.05 \mathrm{~K}$.

The molecular magnetic frame $\left(x^{\prime}, y^{\prime}, z^{\prime}\right)$ was chosen according to the standard system of coordinates for the $\mathrm{N}-\mathrm{O}$ paramagnetic moiety with the $x^{\prime}$ axis along the $\mathrm{N}-\mathrm{O}$ bond ${ }^{31,34}$ and the $z^{\prime}$ axis perpendicular to the five-membered ring, i.e. parallel to the $p_{z}$ orbital containing the unpaired electron. According to a standard approach, the $z$ axis of the molecular ordering frame is considered parallel to the principal axis of inertia of the probe (its "long axis") and, to simplify the rotation which takes the ordering into the magnetic frame ${ }^{31,34}$, the $y$ axis is considered parallel to the $z^{\prime}$ axis. To reduce the correlation among variable parameters, the Euler angles, $\beta$ and $\gamma$, between the molecular frames, were fixed in the fittings to $15^{\circ}$ and $90^{\circ}$, respectively, in agreement with previous results obtained in related systems ${ }^{25,31-34}$.

The EPR spectra simulation program employed was based on a set of Fortran routines implementing the slow tumbling the- 
ory for a spin probe reorienting in a LC, developed by Freed and collaborators $^{24,35,36}$, combined with a software package ${ }^{37}$ that optimizes the fit parameters using the Gauss-Newton-Marquardt non-linear least squares method ${ }^{38}$.

On cooling from the I to the $\mathrm{N}_{\mathrm{u}}$ phase, from $393.2 \mathrm{~K}$, down to $379.2 \mathrm{~K}$, spectra were very well fitted by a simple monodomain aligned along the magnetic field, indicating that the spectrometer field is able to produce an essentially $(\approx 95 \%)$ uniform sample (see the Results and Discussion section for details). In the presence of the magnetic field, this uniformity is expected to be maintained upon the further cooling into the $\mathrm{N}_{\mathrm{tb}}$ phase.

\section{Theory}

The nitroxide spin probe is assumed to reorient in a locally uniaxial nematic domain whose director, $\boldsymbol{n}$, can be oriented, in general, at an angle $\beta_{\mathrm{d}}$ with respect to the spectrometer uniform magnetic field. The corresponding unsaturated, high-field EPR spectrum, $I_{N}\left(\omega-\omega_{0}, \beta_{\mathrm{d}}\right)$, at frequency $\omega$, is calculated using the classic Stochastic Liouville Equation approach of Freed and collaborators ${ }^{24,35,36}$ which predicts that:

$$
I_{N}\left(\omega-\omega_{0}, \beta_{\mathrm{d}}\right)=\frac{1}{\pi}\left\langle\left\langle v\left|\left[(\hat{\boldsymbol{\Gamma}}-i \mathscr{L})+i\left(\omega-\omega_{0}\right) \boldsymbol{I}\right]^{-1}\right| v\right\rangle\right\rangle,
$$

where the central frequency, $\omega_{0}$, at the spectrometer field, $B_{0}$ $(0.339 \mathrm{~T})$, is obtained from the $g$ factor, $g_{0}$, and the Bohr magneton, $\beta_{e}: \omega_{0}=g_{0} \beta_{e} B_{0} / \hbar$. $\mathscr{L}$ is the Liouville superoperator obtained from the orientation dependent spin Hamiltonian, $\hat{\Gamma}$ is the diffusion superoperator describing the reorientational motion of the probe, $|v\rangle$ is a vector containing spin transition moments averaged over the equilibrium ensemble and $\boldsymbol{I}$ is the identity. A brief account of the general approach of Freed and collaborators $24,35,36$ has been presented in a previous work ${ }^{25}$. To reduce the total number of model parameters, the reorientational motion of the probe is assumed to take place in a uniaxial, mean field ordering potential

$$
U(\beta)=-k T\left[\lambda_{20} P_{2}(\cos \beta)\right],
$$

where $\lambda_{20}$ is the strength of the potential, $k$ the Boltzmann constant, $T$ the temperature in Kelvin and $P_{2}(\cos \beta)$ is a second rank Legendre polynomial. The local order is described by the orientational order parameter, $\left\langle P_{2}\right\rangle$, defined as

$$
\left\langle P_{2}\right\rangle=\frac{\int P_{2}(\beta) \exp [-U(\beta) / k T] \sin \beta \mathrm{d} \beta}{\int \exp [-U(\beta) / k T] \sin \beta \mathrm{d} \beta},
$$

where $\beta$ is the probe orientation with respect to the local domain director.

The EPR spectrum of a spin probe in a LC phase, that can be modelled as a distribution of uniaxial nematic domains, is given by the superposition of the monodomain spectra, $I_{N}\left(\omega-\omega_{0}, \beta_{\mathrm{d}}\right)$

$$
I_{\mathrm{T}}\left(\omega-\omega_{0}\right)=\int_{\beta_{\mathrm{d}}} I_{N}\left(\omega-\omega_{0}, \beta_{\mathrm{d}}\right) P\left(\beta_{\mathrm{d}}\right)_{\mathrm{T}} \mathrm{d} \beta_{\mathrm{d}},
$$

where $I_{\mathrm{T}}\left(\omega-\omega_{0}\right)$ is the theoretical EPR spectrum, $P\left(\beta_{\mathrm{d}}\right)_{\mathrm{T}}$ is the distribution of the local nematic director and the integral extends over the entire variation of $\beta_{\mathrm{d}}$.

In the $\mathrm{N}_{\mathrm{tb}}$ phase the local nematic domain director is supposed to precess uniformly about an axis, making a fixed angle, $\theta_{0}$, with it $6,8,10,12,27,39$. A "fixed-tilt" (FT) model can be defined accordingly, indeed, when the axis is oriented along the magnetic field, the local director is uniformly oriented at a fixed angle $\theta_{0}$ and the director distribution is simply a tilted delta function, $P\left(\beta_{\mathrm{d}}\right)_{\mathrm{FT}}=\delta\left(\beta_{\mathrm{d}}-\theta_{0}\right)$.

When the axis is perpendicular to the magnetic field, as indicated in Figure 3, the local director, $\boldsymbol{n}$, is uniformly distributed on the surface of a cone of aperture $2 \theta_{0}$ whose axis is perpendicular to the laboratory $z$ axis, taken as the direction of the magnetic field. In this case, $P\left(\beta_{\mathrm{d}}\right)_{\mathrm{FT}}$ can be obtained as follows.

$\mathrm{A}$ is the projection of $\mathrm{P}$ onto the $\mathrm{xz}$ plane and $\mathrm{B}$ is the projection of $\mathrm{P}$ onto the $\mathrm{z}$ axis. Assuming $\overline{O P}$ unitary, we have: $\overline{O A}=\sin \theta_{0}$ and $\overline{O B}=\cos \phi \sin \theta_{0}$. Since we have also $\overline{O B}=\cos \beta_{\mathrm{d}}, \beta_{\mathrm{d}}$ and $\phi$ can be related as $\cos \beta_{\mathrm{d}}=\cos \phi \sin \theta_{0}$.

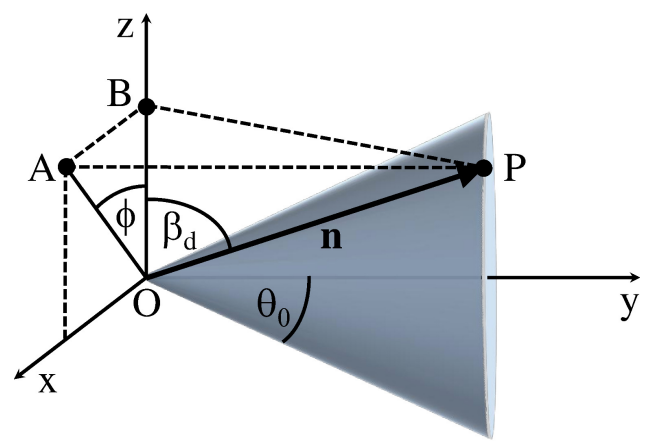

Fig. 3 Schematic of the conical distribution of the local director in the twist-bend model when the cone axis is perpendicular to the $z$ axis, taken as the direction of the magnetic field.

The conical distribution must obey the relation

$$
P\left(\beta_{\mathrm{d}}\right)_{\mathrm{FT}} \mathrm{d} \beta_{\mathrm{d}}=P(\phi) \mathrm{d} \phi
$$

therefore

$$
P\left(\beta_{\mathrm{d}}\right)_{\mathrm{FT}}=P(\phi) \frac{\mathrm{d} \phi}{\mathrm{d} \beta_{\mathrm{d}}}=\frac{1}{2 \pi} \frac{\mathrm{d} \phi}{\mathrm{d} \beta_{\mathrm{d}}},
$$

since the distribution of the angle $\phi$ is uniform. To calculate $\frac{d \phi}{d \beta_{d}}$ we obtain $\phi$ from the previous relation

$$
\phi=\arccos \frac{\cos \beta_{\mathrm{d}}}{\sin \theta_{0}} .
$$

Finally we obtain

$$
\frac{\mathrm{d} \phi}{\mathrm{d} \beta_{\mathrm{d}}}=\frac{\csc \theta_{0} \sin \beta_{\mathrm{d}}}{\sqrt{1-\cos \beta_{\mathrm{d}}^{2} \csc \theta_{0}^{2}}} .
$$

$P\left(\beta_{\mathrm{d}}\right)_{\mathrm{FT}}$ is real for $\frac{\pi}{2}-\theta_{0}<\beta_{\mathrm{d}}<\frac{\pi}{2}+\theta_{0}$. A second rank order parameter, $\left\langle P_{2}\right\rangle_{\mathrm{d}}$, corresponding to this director distribution can be introduced as

$$
\left\langle P_{2}\right\rangle_{\mathrm{d}}=\frac{\int_{\frac{\pi}{2}-\theta_{0}}^{\frac{\pi}{2}+\theta_{0}}\left[\frac{3}{2} \cos ^{2}\left(\beta_{\mathrm{d}}\right)-\frac{1}{2}\right] P\left(\beta_{\mathrm{d}}\right)_{\mathrm{FT}} \mathrm{d} \beta_{\mathrm{d}}}{\int_{\frac{\pi}{2}-\theta_{0}}^{\frac{\pi}{2}+\theta_{0}} P\left(\beta_{\mathrm{d}}\right)_{\mathrm{FT}} \mathrm{d} \beta_{\mathrm{d}}}=\frac{1}{8}\left[-1-3 \cos \left(2 \theta_{0}\right)\right] .
$$

The dependence of the $\left\langle P_{2}\right\rangle_{\mathrm{d}}$ of the distribution upon the angle $\theta_{0}$ shows the expected behaviour. In particular, when $\theta_{0} \rightarrow 0$ the 
distribution reduces to a monodomain perpendicular to the magnetic field and the $\left\langle P_{2}\right\rangle_{\mathrm{d}}$ assumes the limiting value $-1 / 2$; when $\theta_{0} \rightarrow \pi / 2$ the distribution becomes uniform on the $x z$ plane and the limiting value of $\left\langle P_{2}\right\rangle_{\mathrm{d}}$ is $1 / 4$.

As presented in the Results and Discussion section, the analyses of the EPR spectra done with the FT model failed to provide a consistent temperature behaviour of the parameters recovered from the fits. The main problem with the FT model seemed to be the following. When the axis of the $\mathrm{N}_{\mathrm{tb}}$ phase is parallel to the magnetic field, the EPR spectrum should be just a tilted monodomain, since the local domain director twists around the axis with a constant tilt angle $\theta_{0}$. Instead, the experimental spectra in the $\mathrm{N}_{\mathrm{tb}}$ phase were clearly of the polydomain type.

A conceivable model that maintains the basic structure of the twist-bend phase and is also compatible with polydomain EPR spectra is one in which the local domain director is not fixed at $\theta_{0}$ with respect to the magnetic field but is described by a relatively narrow distribution, centred at $\theta_{0}$. The relevant angular distribution for this "distributed-tilt" (DT) model has been obtained as follows.

The uniform magnetic field is oriented along the $z$ axis of the lab reference frame $x, y, z$ (Figure 4). The uniform tilt direction of the twist-bend phase is defined by the $z^{\prime}$ axis of a second reference frame, $x^{\prime}, y^{\prime}, z^{\prime}$, rotated with respect to $x, y, z$ by the Euler angles $0, \theta_{0}, 0$. The local domain director, $\boldsymbol{n}$, is taken as a unitary vector having, in the $x^{\prime}, y^{\prime}, z^{\prime}$ frame, coordinates $\sin \phi \cos \alpha, \sin \phi \sin \alpha, \cos \phi$. The angle between the director, $\boldsymbol{n}$, and the magnetic field is $\beta_{\mathrm{d}}$.
The unknown probability density distribution of the director, $\boldsymbol{n}$, in the $x^{\prime}, y^{\prime}, z^{\prime}$ frame, is assumed to be uniaxial, centred on the $z^{\prime}$ axis, with a uniform distribution of $\alpha, P(\alpha)=1 / 2 \pi$, whereas the distribution of the polar angle $\phi$ is modelled, as it is usually done (e.g. in the EPRLL "family" of EPR spectra simulation programs ${ }^{40}$ ) by a $P_{2}$-type distribution, $P(\phi) \propto \exp \left[\lambda_{\mathrm{d}} P_{2}(\cos \phi)\right]$, where $P_{2}$ is a second rank Legendre polynomial and $\lambda_{\mathrm{d}}$ is a positive constant.

The order of the domain director with respect to $z^{\prime}$ is described by the orientational order parameter, $\left\langle P_{2}\right\rangle_{\mathrm{d}}$, defined as

$$
\left\langle P_{2}\right\rangle_{\mathrm{d}}=\frac{\int P_{2}(\cos \phi) \exp \left[\lambda_{\mathrm{d}} P_{2}(\cos \phi)\right] \sin \phi \mathrm{d} \phi}{\int \exp \left[\lambda_{\mathrm{d}} P_{2}(\cos \phi)\right] \sin \phi \mathrm{d} \phi} .
$$

A value of $\left\langle P_{2}\right\rangle_{\mathrm{d}}=0$ represents the limiting case of an isotropic distribution of the domain directors, corresponding to $P\left(\beta_{\mathrm{d}}\right)_{\mathrm{DT}}=$ $1 / \pi$, whereas in the other limit of $\left\langle P_{2}\right\rangle_{\mathrm{d}}=1$, the local director is uniformly oriented at a fixed angle $\theta_{0}$ and the director distribution becomes identical to the previous model, $P\left(\beta_{\mathrm{d}}\right)_{\mathrm{DT}}=$ $P\left(\beta_{\mathrm{d}}\right)_{\mathrm{FT}}=\delta\left(\beta_{\mathrm{d}}-\theta_{0}\right)$.

An explicit equation for the probability density distribution of the angle $\beta_{\mathrm{d}}$ can be obtained by expressing $\phi$ in terms of $\beta_{\mathrm{d}}$ by noticing that $\cos \beta_{\mathrm{d}}$ and $\cos \phi$ are the $z$ and $z^{\prime}$ coordinates of $\boldsymbol{n}$, respectively (see Figure 4), related by

$$
\cos \beta_{\mathrm{d}}=\sin \theta_{0} \sin \phi \cos \alpha+\cos \theta_{0} \cos \phi .
$$

The expression of $\phi$ in terms of $\beta_{\mathrm{d}}$ is obtained, after some algebra, as

$$
\cos ^{2} \phi=f\left(\alpha, \beta_{\mathrm{d}}\right)=\frac{\sec ^{2} \theta_{0}\left(\cos \beta_{\mathrm{d}}-\cos \alpha \tan \theta_{0} \sqrt{\cos ^{2} \alpha \sin ^{2} \theta_{0}-\cos ^{2} \beta_{\mathrm{d}}+\cos ^{2} \theta_{0}}\right)^{2}}{\left(1+\cos ^{2} \alpha \tan ^{2} \theta_{0}\right)^{2}}
$$

The probability density distribution of the angle $\beta_{\mathrm{d}}$ is therefore

$$
P\left(\alpha, \beta_{\mathrm{d}}\right)_{\mathrm{DT}}=\exp \left[\lambda_{\mathrm{d}}\left(\frac{3}{2} f\left(\alpha, \beta_{\mathrm{d}}\right)-\frac{1}{2}\right)\right] .
$$

The integration of this expression along both $\alpha$ and $\beta_{\mathrm{d}}$ is carried out numerically using the adaptive multidimensional integration Fortran routine DCUHRE ${ }^{41}$.

It is worth pointing out that such a distribution of tilt angles with respect to the magnetic field is not necessarily the only one that could fit the spectra. The reason is that a magnetic resonance experiment like our one is only sensitive to the angle between a molecular magnetic tensor axis and the spectrometer magnetic field direction. It is conceivable that another distribution could, for example, describe a situation in which the helical axis, although parallel on average to the magnetic field, is undulating while the tilt angle is fixed with respect to the helix axis ${ }^{42}$. However, yet another possibility is that of a more inhomogeneous distribution of fluctuations of the helix axis as well as of tilt angles. Any model of this type would, however, require additional fitting parameters and, we believe, its introduction would not be justi- fied by our current observable results. We thus stick, following Occam's razor principle, to the minimal deviation from the standard heliconical model that fits our spectra.

\section{Results and Discussion}

The ability of the CSL spin probe to report correctly the local nematic order of the CB11CB LC can be assessed by noticing that CSL was able to consistently report the local order and the director organization of the 5CB LC in previous studies ${ }^{43,44}$. It has also been shown by Oganesyan et al., using molecular dynamics simulations of the CSL probe in $5 \mathrm{CB}^{45}$, that the order parameter of the probe and of the mesogen are in good agreement. If the local environment in which the CSL spin probe is located is similar in these two different but related LCs, it is reasonable to expect that CSL will report correctly the local nematic order also of the CB11CB LC.

In Figure 5 a typical rigid-limit spectrum of $\mathrm{CB} 11 \mathrm{CB}$, recorded in the bulk, is compared with typical rigid-limit bulk spectra of $1^{\prime \prime}, 9^{\prime \prime}$-bis(4-cyanobiphenyl-4'-yl)nonane (CB9CB) and of 5CB. For every LC the spectrum was recorded at a temperature well be- 




Fig. 4 Schematic of the geometrical relation between $\cos \left(\beta_{\mathrm{d}}\right)$ and $\cos (\phi)$ as the $z$ and $z^{\prime}$ coordinates of the local domain director $\boldsymbol{n}$ (see text for details).

low the relative melting temperature, $T_{\mathrm{m}}$, and in the rigid-limit regime. The resulting reduced temperatures, $T^{*} \equiv T / T_{\mathrm{m}}$, were thus 0.733 for $\mathrm{CB} 11 \mathrm{CB}, 0.616$ for $\mathrm{CB} 9 \mathrm{CB}$ and 0.586 for $5 \mathrm{CB}$. Melting temperatures, observed on heating, were $377.2 \mathrm{~K}$ for $\mathrm{CB} 11 \mathrm{CB}$, $362.15 \mathrm{~K}$ for $\mathrm{CB} 9 \mathrm{CB}$ and $295.7 \mathrm{~K}$ for 5CB. Apart from a small difference in the linewidths, the position of the peaks, which is determined solely by the hyperfine tensor which, in turn, is dependent on the polarity of the environment, is the same in the three LCs indicating that (i) the rigid-limit spectrum of CB11CB is typical of these kind of dimeric LCs and that (ii) the local environment in which the CSL spin probe is located should be fairly similar in 5CB and in CB11CB.

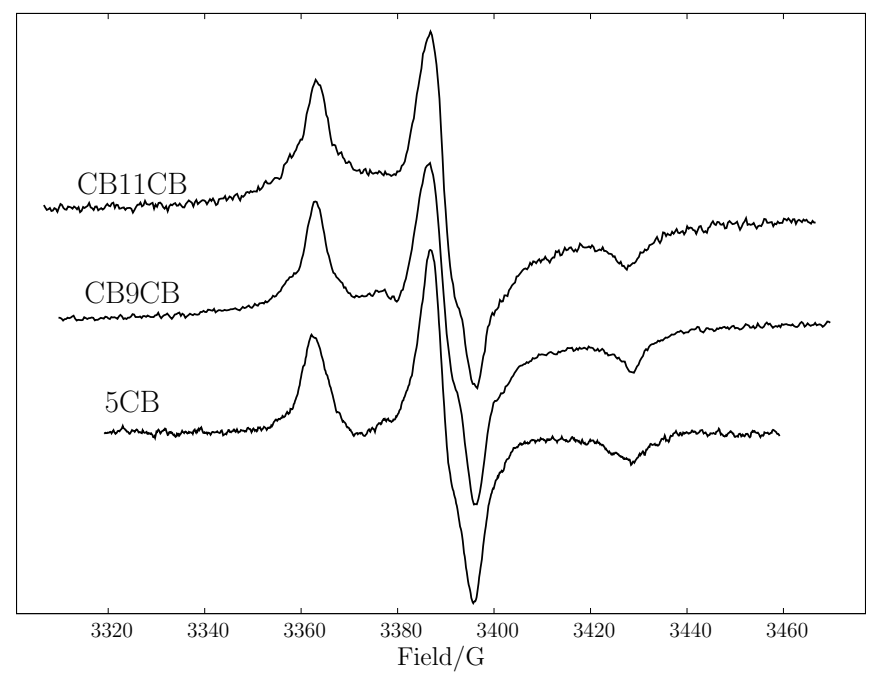

Fig. 5 Typical rigid-limit spectrum of $\mathrm{CB} 11 \mathrm{CB}$, recorded in the bulk at the reduced temperature, $T^{*} \equiv T / T_{\mathrm{m}}$, of 0.733 , compared with a typical rigid-limit bulk spectrum of $\mathrm{CB} 9 \mathrm{CB}$ at $T^{*}=0.616$ and with a typical rigid-limit bulk spectrum of $5 \mathrm{CB}$ at $T^{*}=0.586 . T_{\mathrm{m}}$ is the melting temperature observed on heating which is $377.2 \mathrm{~K}$ for $\mathrm{CB} 11 \mathrm{CB}, 362.15$ $\mathrm{K}$ for $\mathrm{CB} 9 \mathrm{CB}$ and $295.7 \mathrm{~K}$ for $5 \mathrm{CB}$.

Final EPR spectra were recorded, on cooling, in the temperature interval $403.2-368.2 \mathrm{~K}$ to study the isotropic and the nematic phases (see below) exhibited by the CB11CB LC. EPR spectra recorded between 403.2 and $395.2 \mathrm{~K}$ had three well-resolved hyperfine lines typical of a relatively fast motional isotropic regime, without other contributions, clearly indicating that the $\mathrm{CB} 11 \mathrm{CB}$ was in the I phase. These spectra were consistently fitted to the isotropic model. Two spectra typical of the I phase at 403.2 and $395.2 \mathrm{~K}$ are shown in Figure 6 (top spectra, black line) together with the corresponding fits (red line).

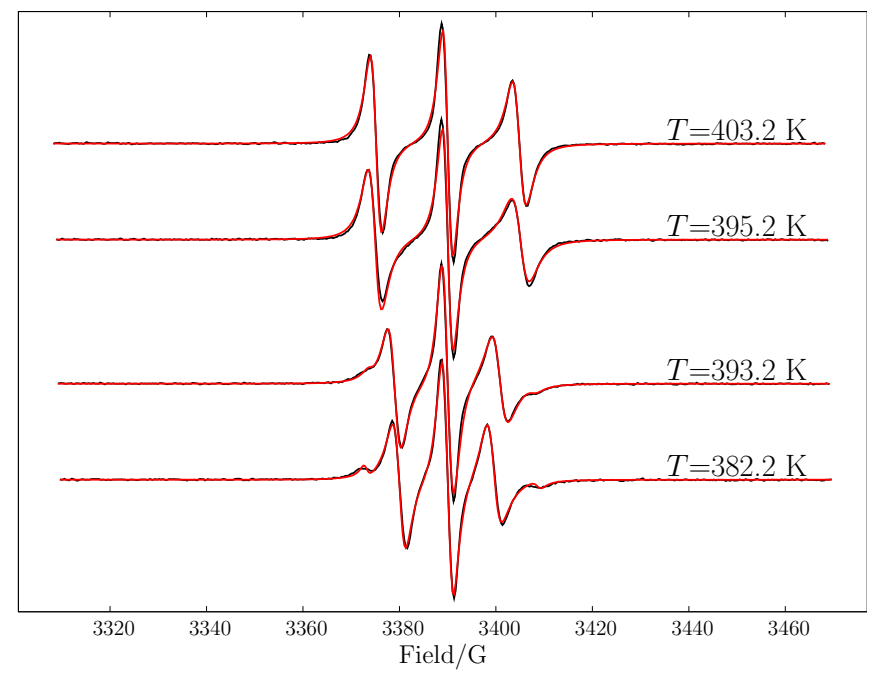

Fig. 6 Typical EPR spectra (black line) in the I phase (top two spectra) and in the $\mathrm{N}_{\mathrm{u}}$ phase (bottom two spectra). Top spectra are fitted (red line) to an isotropic model; bottom spectra are fitted to a nematic monodomain model with a small contribution (about $5 \%$ ) of an isotropic distribution of domains (see text for details).

By lowering the temperature at $393.2 \mathrm{~K}$, the position of the outermost peaks, previously constant, suddenly changed, indicating that a phase transition had occurred. The approximate temperature of this transition was $394.2 \mathrm{~K}$, about $4 \mathrm{~K}$ below the $T_{\mathrm{NI}}$ of CB11CB not doped with CSL, measured via DSC on heating. A downshift of the transition temperature of a few $\mathrm{K}$ has been observed in previous works on the related $\mathrm{LC} C \mathrm{CB}^{2} \mathrm{CB}^{5,46}$ in presence of a small amount of a dopant. The observed spectral lineshapes were typical of a $\mathrm{N}_{\mathrm{u}}$ phase, as expected, with the director uniformly aligned along the magnetic field. Two spectra typical of the $\mathrm{N}_{\mathrm{u}}$ phase at 393.2 and $382.2 \mathrm{~K}$ are shown in Figure 6 (bottom spectra, black line). A very small contribution, determined in the analysis to be about $5 \%$, can be seen as shoulders or peaks at the extremes of the main spectrum. In the temperature interval 393.2-379.2 $\mathrm{K}$, the main spectrum was modelled as a uniaxial nematic monodomain obtaining a consistent temperature dependence of the best-fit parameters. The small outer peaks were not compatible with the isotropic model and were approximately reproduced with the DT model, with parameters $\left\langle P_{2}\right\rangle_{\mathrm{d}}=0$ and tilt angle $\theta_{0}=0^{\circ}$, which corresponds to an isotropic distribution of domains. The nature of this contribution, being quite small, could not be determined more accurately. Fits of the spectra at 393.2 and $382.2 \mathrm{~K}$ are also shown in Figure 6 (bottom spectra, red line).

In a series of preliminary analyses, spectra in the range $378.2-$ $376.7 \mathrm{~K}$ showed a progressively lower uniaxial nematic spectral contribution and a new, increasing, contribution. This behaviour 
suggested the presence in our CSL-doped CB11CB LC of a biphasic region in which the $\mathrm{N}_{\mathrm{u}}$ and the $\mathrm{N}_{\mathrm{tb}}$ phases coexist over a temperature interval of a few $\mathrm{K}$. A coexistence region, induced by a small amount of a dopant, was indeed observed in previous works on the related $\mathrm{LC}$ CB7CB ${ }^{5,46}$. This coexistence is consistent with the first order nature of the $\mathrm{N}_{\mathrm{tb}}-\mathrm{N}_{\mathrm{u}}$ transition in these materials, as reported by Tripathi et el. ${ }^{47}$ for the dimer homologue CB9CB. Below $376.7 \mathrm{~K}$ the uniaxial nematic spectral contribution was no longer present, suggesting that the system was fully in the lower temperature $\mathrm{N}_{\mathrm{tb}}$ phase. In this phase, spectra showed additional hyperfine contributions at low and high field, indicating a nonuniform alignment of the director with respect to the magnetic field. A similar behaviour has been found by Cestari et al. ${ }^{2}$ who recorded a series of EPR spectra across the $\mathrm{N}_{\mathrm{u}}$ and $\mathrm{N}_{\mathrm{tb}}$ phases of CB7CB.

Before any attempt to analyse the spectra in the coexistence region could be made, it was necessary to model the spectra in the lower temperature $\mathrm{N}_{\mathrm{tb}}$ phase.

Spectra recorded between 375.7 and $372.2 \mathrm{~K}$ were quite similar, thus confirming that the system was not experiencing further phase changes. The spectrum recorded at $372.7 \mathrm{~K}$ was therefore considered as representative of the $\mathrm{N}_{\mathrm{tb}}$ phase to be compared against the FT model. The theoretical spectrum was calculated by fixing the FT model parameters at the following values: the local order parameter has been shown ${ }^{2,5,27}$ to increase slightly upon entering the $\mathrm{N}_{\mathrm{tb}}$ phase, accordingly, $\left\langle P_{2}\right\rangle$ was set at 0.50 which is slightly larger than the value of 0.49 found at the lower end of the $\mathrm{N}_{\mathrm{u}}$ phase at $379.2 \mathrm{~K}$; the $\mathrm{N}_{\mathrm{tb}}$ phase of CB11CB was found to be more viscous than the $\mathrm{N}_{\mathrm{u}}$ phase ${ }^{28}$, accordingly, to explore a wider dynamical range, the perpendicular component of the rotational diffusion tensor, $D_{\perp}$, was set at a series of different values between $0.01 \mathrm{~ns}^{-1}$ and $0.002 \mathrm{~ns}^{-1}$, which were either half or one tenth of the value of $0.02 \mathrm{~ns}^{-1}$ observed at the lower end of the $\mathrm{N}_{\mathrm{u}}$ phase, respectively. An estimate of the conical angle, $\theta_{0}$, for $\mathrm{CB} 11 \mathrm{CB}$ has not been reported. The closest available estimate is the conical angle for CB7CB that has been recently determined from the mean value of the ${ }^{2} \mathrm{H}$ NMR quadrupole splittings of CB7CB- $\mathrm{d}_{4}$ and $8 C B-\mathrm{d}_{2}$ dissolved in $\mathrm{CB} \mathrm{CB}^{27}$, from optical birefringence measurements ${ }^{12}$ and using transmission electron microscopy $^{8}$. In a range of a few $\mathrm{K}$ below the $\mathrm{N}_{\mathrm{tb}}-\mathrm{N}_{\mathrm{u}}$ transition all these studies determined $\theta_{0}$ to be about $20^{\circ}$. A different estimate of the conical angle for $\mathrm{CB} 7 \mathrm{CB}$, also from optical birefringence measurements ${ }^{42}$, determined $\theta_{0}$ to be about $10^{\circ}$. Following these arguments and to explore a wider range of values, $\theta_{0}$ was set either at $10^{\circ}$ or at $30^{\circ}$.

In Figure 7, top, the EPR spectrum (black line) at $372.7 \mathrm{~K}$ in the $\mathrm{N}_{\mathrm{tb}}$ phase is compared with spectra calculated according to the FT model, with $\left\langle P_{2}\right\rangle=0.50, D_{\perp}=0.01 \mathrm{~ns}^{-1}$ (lower values did not improve the model) and $\theta_{0}=10^{\circ}$ (red line) or $\theta_{0}=30^{\circ}$ (green line). The comparison clearly shows that the lineshapes obtained with the FT model are quite different from the experimental spectrum.

To better understand the nature of the director distribution in the $\mathrm{N}_{\mathrm{tb}}$ phase, spectra at selected temperatures were recorded immediately after "field cooling" (FC) which was done by heating the sample above its $T_{\mathrm{NI}}$ then slowly cooling it to the requested temperature at approximately $1 \mathrm{~K} / \mathrm{min}$, with the applied magnetic field set at $0.63 \mathrm{~T}$, the highest available on the EPR spectrometer. This procedure should maximise the alignment of the local nematic directors along the magnetic field. A second spectrum was then recorded immediately after a rotation of the sample by $90^{\circ}$ around an axis perpendicular to the magnetic field.

In Figure 7, middle and bottom, the EPR spectra (black line) recorded at $370.2 \mathrm{~K}$ in the supercooled $\mathrm{N}_{\mathrm{tb}}$ phase after $\mathrm{FC}(\|)$ and after a $90^{\circ}$ rotation of the sample $(\perp)$ are presented. At this temperature spectra before and after the rotation were, as expected, clearly different, since the phase is anisotropic and, due to the increased viscosity, the relatively low magnetic field used in the measurements (centred at $0.339 \mathrm{~T}$ ) was not able to realign the sample. In fact, spectra recorded immediately after the rotation and several hours later were identical (not shown). Since the $\mathrm{N}_{\mathrm{tb}}$ phase of CB11CB in a standard LC cell was not switched by an electric field lower than $20 \mathrm{~V} / \mu \mathrm{m},{ }^{9}$ it seems unlikely that the director distribution will be perturbed by the relatively weak magnetic field of the EPR spectrometer.

These spectra were then compared against the FT model. The theoretical spectra were calculated by fixing the model parameters at the following values: since it has been shown ${ }^{2,5,27}$ that, once in the $\mathrm{N}_{\mathrm{tb}}$ phase, the order parameter $\left\langle P_{2}\right\rangle$ remains almost constant, it was set at the same previous value of 0.50 ; since the temperature was only $2.5 \mathrm{~K}$ lower than the previous one, the rotational diffusion tensor, $D_{\perp}$, was also set at the same value of $0.01 \mathrm{~ns}^{-1}$; the conical angle, $\theta_{0}$, was set, as before, either at $10^{\circ}$ or at $30^{\circ}$. This interval should still be appropriate to model the tilt angle that has been reported to maintain a value within $30^{\circ}$ in a range of temperatures not too far from the $\mathrm{N}_{\mathrm{tb}}-\mathrm{N}_{\mathrm{u}}$ transition $8,12,27$.

In Figure 7, middle and bottom, the EPR spectra (black line) at $370.2 \mathrm{~K}$ in the supercooled $\mathrm{N}_{\mathrm{tb}}$ phase are compared to spectra calculated according to the FT model, with parameter values described above and $\theta_{0}=10^{\circ}$ (red line) or $\theta_{0}=30^{\circ}$ (green line). We see that the FT model is able to qualitatively reproduce the spectrum recorded after the rotation $(\perp)$, but not the spectrum recorded before the rotation $(\|)$.

Despite the lack of agreement of spectra calculated according to the FT model with experimental EPR spectra at selected temperatures, an attempt was done to analyse spectra with the FT model, by optimizing fit parameters, from the onset of the biphasic region, at $T=378.2 \mathrm{~K}$, to $T=368.2 \mathrm{~K}$, in the supercooled $\mathrm{N}_{\mathrm{tb}}$ phase. To reduce the number of model parameters, different globalization schemes were adopted where the tilt angle, $\theta_{0}$, was kept variable but was a common parameter to model a group of spectra. In all cases, parameters recovered from the fits failed to show a consistent and meaningful behaviour with temperature, indicating, as expected, that the FT model was not appropriate to correctly reproduce the experimental spectra.

The DT model, presented in the Theory section, was therefore tested against the experimental EPR spectra recorded at $372.7 \mathrm{~K}$ in the $\mathrm{N}_{\mathrm{tb}}$ phase and at $370.2 \mathrm{~K}$ in the supercooled $\mathrm{N}_{\mathrm{tb}}$ phase. In Figure 8, top, the EPR spectrum (black line) at $372.7 \mathrm{~K}$ in the $\mathrm{N}_{\text {tb }}$ phase is compared with spectra calculated according to the DT model, with $\left\langle P_{2}\right\rangle=0.50,\left\langle P_{2}\right\rangle_{\mathrm{d}}=0.44, D_{\perp}=0.01 \mathrm{~ns}^{-1}$ and $\theta_{0}=10^{\circ}$ (red line) or $\theta_{0}=30^{\circ}$ (green line). In the same Figure, middle and 


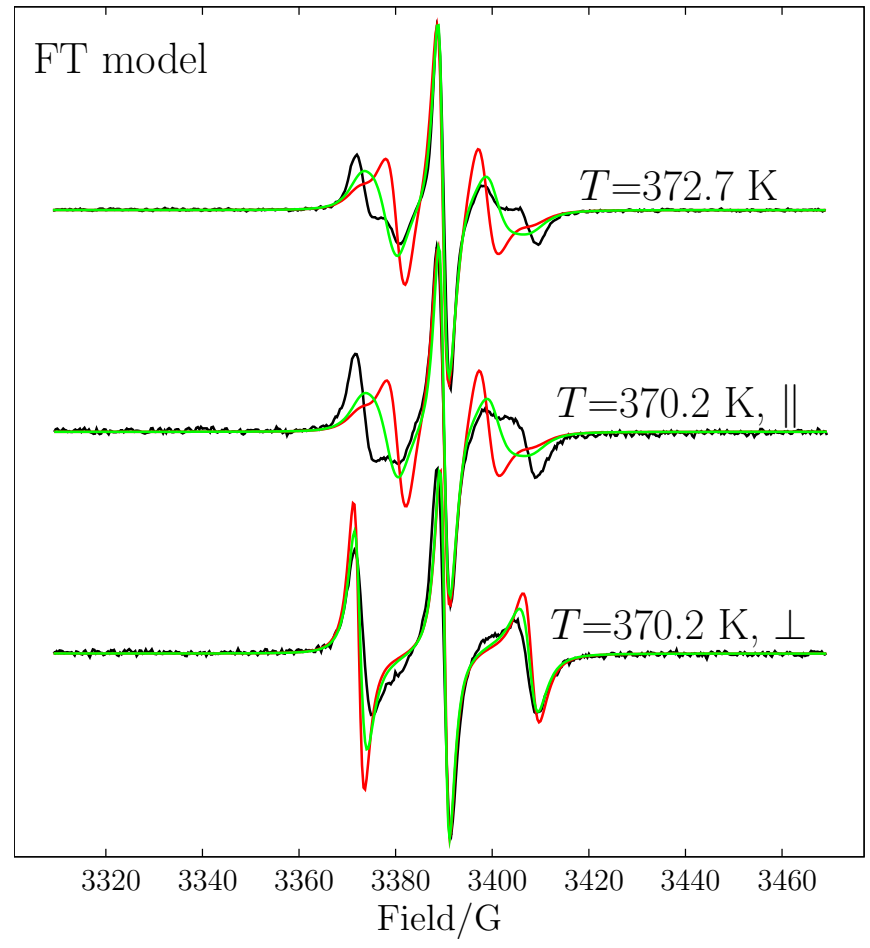

Fig. 7 EPR spectra (black line) at $372.7 \mathrm{~K}$ in the $\mathrm{N}_{\mathrm{tb}}$ phase, top, or at $370.2 \mathrm{~K}$ in the supercooled $\mathrm{N}_{\mathrm{tb}}$ phase, middle and bottom, after $\mathrm{FC}(\|)$ and after a $90^{\circ}$ rotation of the sample around an axis perpendicular to the magnetic field $(\perp)$, compared to spectra calculated according to the FT model with tilt angle $\theta_{0}=10^{\circ}$ (red line) or $\theta_{0}=30^{\circ}$ (green line, see text for details).

bottom, the EPR spectra (black line) at $370.2 \mathrm{~K}$ in the supercooled $\mathrm{N}_{\mathrm{tb}}$ phase are compared to spectra calculated according to the DT model, with $\left\langle P_{2}\right\rangle=0.50,\left\langle P_{2}\right\rangle_{\mathrm{d}}=0.40, D_{\perp}=0.01 \mathrm{~ns}^{-1}$ and $\theta_{0}=$ $10^{\circ}$ (red line) or $\theta_{0}=30^{\circ}$ (green line). It is apparent that the lineshapes of the spectra calculated with the DT model resemble more closely those of the experimental spectra compared to the lineshapes of the spectra calculated with the FT model shown in Figure 7.

Experimental spectra recorded in the temperature interval from the onset of the biphasic region, at $T=378.2 \mathrm{~K}$, to $T=368.2 \mathrm{~K}$, in the supercooled $\mathrm{N}_{\mathrm{tb}}$ phase, were analysed with the DT model. To reduce the correlation among the fit parameters, separate global analyses were performed for the spectra recorded in the biphasic region, those in the $\mathrm{N}_{\mathrm{tb}}$ phase and those in the supercooled $\mathrm{N}_{\mathrm{tb}}$ phase. In each global analysis, the tilt angle, $\theta_{0}$, and the order parameter of the domain directors, $\left\langle P_{2}\right\rangle_{\mathrm{d}}$, were global parameters.

With this approach, parameters recovered from the fits appeared to have a variation that was consistent across the biphasic region, the $\mathrm{N}_{\mathrm{tb}}$ and the supercooled $\mathrm{N}_{\mathrm{tb}}$ phases and was also consistent with the parameters previously recovered from the I and the $\mathrm{N}_{\mathrm{u}}$ phases. The temperature dependence of the local orientational order parameter, $\left\langle P_{2}\right\rangle$, and of the rotational diffusion tensor, $D_{\perp}$, across the whole temperature interval studied is shown in Figures 9 and 10, respectively. Recovered global parameters were as follows, biphasic region: $\theta_{0}=16^{\circ},\left\langle P_{2}\right\rangle_{\mathrm{d}}=0.60 ; \mathrm{N}_{\mathrm{tb}}$ phase: $\theta_{0}=11^{\circ},\left\langle P_{2}\right\rangle_{\mathrm{d}}=0.47$; supercooled $\mathrm{N}_{\mathrm{tb}}$ phase: $\theta_{0}=20^{\circ}$,

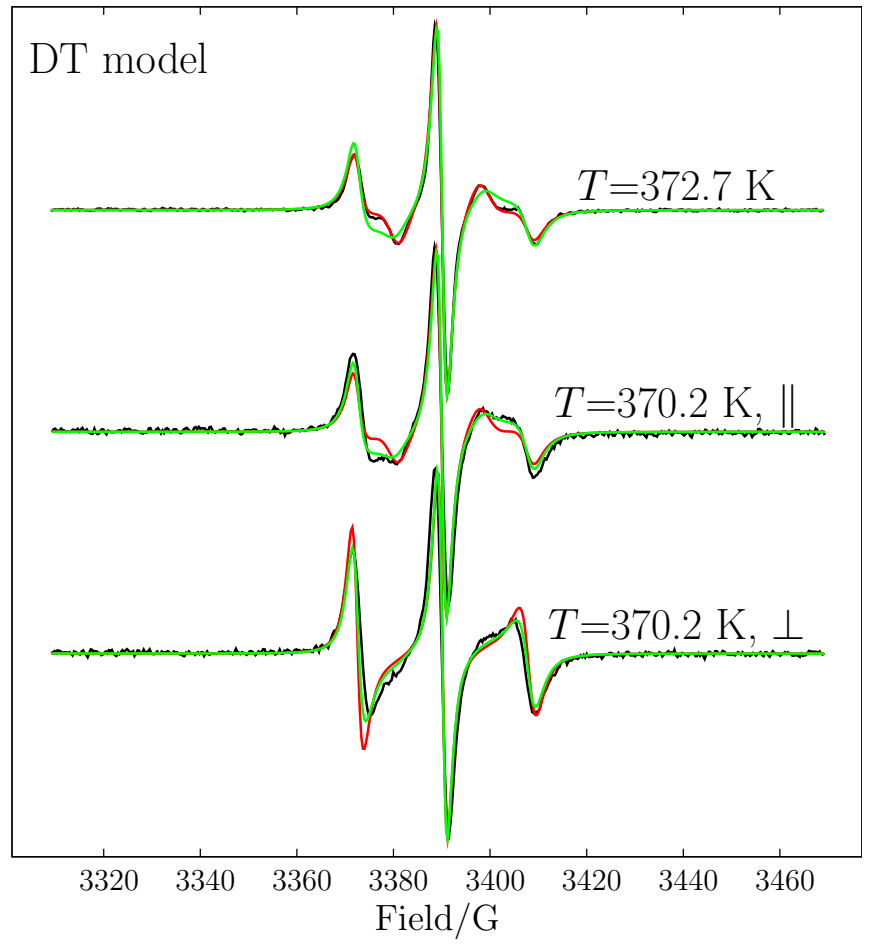

Fig. 8 EPR spectra (black line) at $372.7 \mathrm{~K}$ in the $\mathrm{N}_{\mathrm{tb}}$ phase, top, or at $370.2 \mathrm{~K}$ in the supercooled $\mathrm{N}_{\mathrm{tb}}$ phase, middle and bottom, after $\mathrm{FC}(\|)$ and after $a 90^{\circ}$ rotation of the sample around an axis perpendicular to the magnetic field $(\perp)$, compared to spectra calculated according to the distributed-tilt (DT) model with tilt angle $\theta_{0}=10^{\circ}$ (red line) or $\theta_{0}=30^{\circ}$ (green line, see text for details).

$\left\langle P_{2}\right\rangle_{\mathrm{d}}=0.57$.

At the $\mathrm{N}_{\mathrm{tb}}-\mathrm{N}_{\mathrm{u}}$ phase transition, the $\left\langle P_{2}\right\rangle$ values in the coexistence region (two values on one side of the transition and two on the other) show a very small discontinuity which is within the experimental error, therefore the variation of the $\left\langle P_{2}\right\rangle$ with the temperature is essentially continuous. This behaviour appears to be in agreement with the theoretical approach of Dozov ${ }^{4}$ and the molecular theory proposed by Greco et al. ${ }^{16}$.

The observed $\left\langle P_{2}\right\rangle$ values are also in good agreement with those recently obtained by Zhang et al. from polarized Raman spectroscopy in $\mathrm{CB}_{11 \mathrm{CB}^{48}}$ across the $\mathrm{N}_{\mathrm{u}}$ phase and immediately below the $\mathrm{N}_{\mathrm{tb}}-\mathrm{N}_{\mathrm{u}}$ phase transition. In particular, Zhang et al. observed, as in our study, that the local $\left\langle P_{2}\right\rangle$ appears to be continuous across the $\mathrm{N}_{\mathrm{tb}}-\mathrm{N}_{\mathrm{u}}$ phase transition and then remains essentially constant in the $\mathrm{N}_{\mathrm{tb}}$ phase.

In the supercooled $\mathrm{N}_{\mathrm{tb}}$ phase, $\left\langle P_{2}\right\rangle$ values appeared to decrease (see Figure 9, open squares). This was not observed in previous studies on the related dimers $\mathrm{CB}_{7 \mathrm{CB}}{ }^{27}$ and $\mathrm{CB} 9 \mathrm{CB}^{20}$. A similar behaviour has been observed by Emsley et al. in a recent study on the symmetric difluoroterphenyl dimer DTC5C9 ${ }^{49}$. The behaviour of the $\left\langle P_{2}\right\rangle$ in our study bears another similarity with that observed by Emsley et al. in that it reaches a maximum value in the $\mathrm{N}_{\mathrm{u}}$ phase, before the transition to the $\mathrm{N}_{\mathrm{tb}}$ phase. The decrease of $\left\langle P_{2}\right\rangle$ values in the $\mathrm{N}_{\mathrm{tb}}$ phase observed by Emsley et al. was explained as an effect of the simultaneous increase of the tilt angle, $\theta_{0}$. 
Indeed, in our fits, a certain degree of correlation was present between the local $\left\langle P_{2}\right\rangle$ parameter and the tilt angle, $\theta_{0}$ and to study this effect, we repeated the spectral fits by assuming that the $\left\langle P_{2}\right\rangle$ in the supercooled $\mathrm{N}_{\mathrm{tb}}$ phase had an empirical Hallertype ${ }^{50}$ temperature dependence described as

$$
\left\langle P_{2}\right\rangle(T)=\left\langle P_{2}\right\rangle_{0}\left(1-T / T_{N I}\right)^{\beta},
$$

where $T_{N I}$ was fixed at $394.2 \mathrm{~K}$, the temperature of the observed $\mathrm{N}_{\mathrm{u}}-\mathrm{I}$ transition, whereas $\left\langle P_{2}\right\rangle_{0}$ and the exponent, $\beta$, were obtained by fitting the Haller equation 14 to the $\left\langle P_{2}\right\rangle$ values in the $\mathrm{N}_{\mathrm{u}}$ phase in the range from $T_{N I}$ up to the observed maximum around $383 \mathrm{~K}$ (first 7 values, see Figure 9). Best-fit parameters were $\left\langle P_{2}\right\rangle_{0}=0.70$ and $\beta=0.09$. The corresponding Haller function is shown as a dashed line in Figure 9.

Best-fit values of the tilt angle, $\theta_{0}$, recovered from the analysis of the EPR spectra in which the local $\left\langle P_{2}\right\rangle$ was fixed at the Haller values are shown in the inset of Figure 9 (solid circles). These values of the tilt angle are clearly larger than the value of $20^{\circ}$ recovered from previous fits, where $\theta_{0}$ was treated as a global parameter (see above). The quality of these new fits appears to be slightly lower with respect to the previous ones. As an example, the fit of the spectrum at $T=371.7 \mathrm{~K}$, in the supercooled $\mathrm{N}_{\mathrm{tb}}$ phase, is shown in Figure 11. We notice, however, that a similar increase of the tilt angle upon lowering the temperature in the $\mathrm{N}_{\mathrm{tb}}$ was also reported for CB7CB from birefringence measurements ${ }^{51}$.

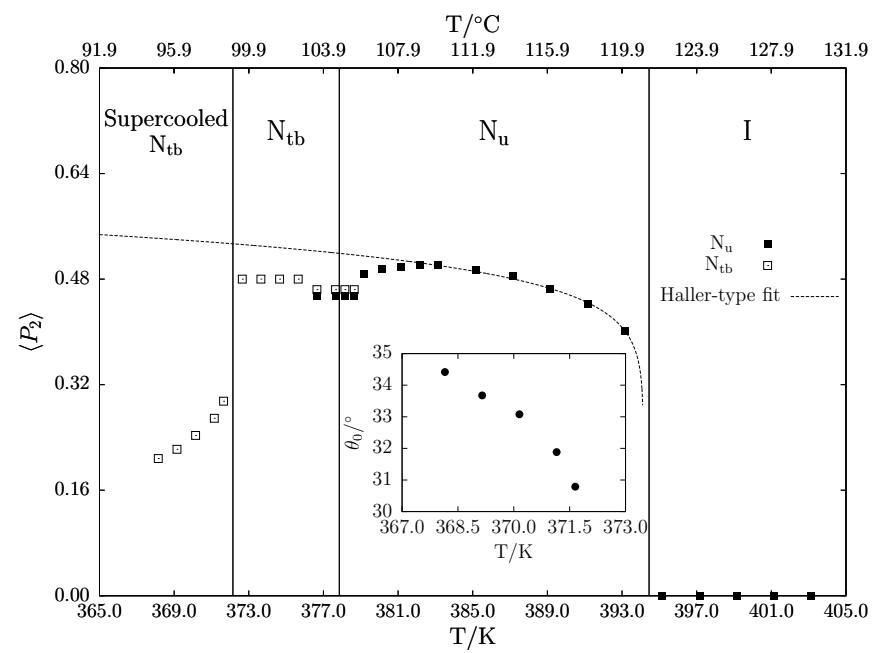

Fig. 9 Temperature dependence of the local orientational order parameter, $\left\langle P_{2}\right\rangle$, in the I and $\mathrm{N}_{\mathrm{u}}$ phase (solid squares) and in the $\mathrm{N}_{\mathrm{tb}}$ and supercooled $\mathrm{N}_{\mathrm{tb}}$ phase (open squares), compared with values calculated with the Haller function of eqn. 14 (dashed line, see text for details). The inset shows the temperature dependence of the tilt angle, $\theta_{0}$ (solid circles), in the supercooled $\mathrm{N}_{\mathrm{tb}}$ phase recovered from the EPR fits, having fixed the $\left\langle P_{2}\right\rangle$ at the Haller values. Vertical lines indicate the approximate transition temperatures observed, that are about $4 \mathrm{~K}$ below the transitions of CB11CB not doped with CSL, measured via DSC on heating. Four temperature values are in the biphasic region: two on one side of the $\mathrm{N}_{\mathrm{tb}}-\mathrm{N}_{\mathrm{u}}$ phase transition and two on the other.

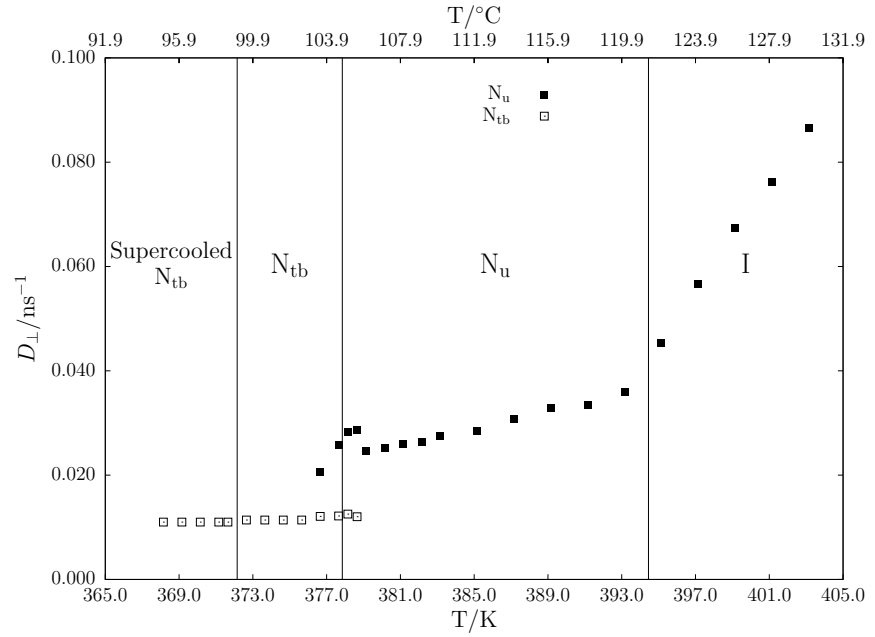

Fig. 10 Temperature dependence of the rotational diffusion tensor, $D_{\perp}$, in the I and $\mathrm{N}_{\mathrm{u}}$ phase (solid squares) and in the $\mathrm{N}_{\mathrm{tb}}$ and supercooled $\mathrm{N}_{\mathrm{tb}}$ phase (open squares). Vertical lines indicate the approximate transition temperatures observed, that are about $4 \mathrm{~K}$ below the transitions of CB11CB not doped with CSL, measured via DSC on heating. Four temperature values are in the biphasic region: two on one side of the $\mathrm{N}_{\mathrm{tb}}-\mathrm{N}_{\mathrm{u}}$ phase transition and two on the other (see text for details).

\section{Conclusions}

EPR spectra of the CSL spin probe dissolved in the nematic phases exhibited by the CB11CB liquid crystal confirmed the presence of an higher temperature uniaxial nematic phase and of a lower temperature nematic phase in which the director distribution is not uniform. This lower temperature nematic phase has been recently described as a twist-bend phase $6,8,10,12,27,39$ where the local nematic domain director precesses uniformly about an axis, making a fixed angle, $\theta_{0}$, with it. The EPR spin probe technique is particularly suitable to examine the differences between the orientational order $\left\langle P_{2}\right\rangle$ with respect to a local director as well as the distribution of local domains, characterized by a director order parameter $\left\langle P_{2}\right\rangle_{\mathrm{d}}$. This could be particularly important when $\left\langle P_{2}\right\rangle_{\mathrm{d}}$ is very different from one, i.e. the sample is not a monodomain. The limiting case is that of an isotropic distributions of domains, like in a spherical membrane vesicle ${ }^{52}$ or in a macroscopically isotropic LC elastomer ${ }^{53}$, where the local domains are ordered, but the overall macroscopic order in the laboratory frame is vanishing. Thus the EPR spin probe method has some advantages on techniques like birefringence that measure the overall order of the sample in the laboratory frame. Moreover, the magnetic field employed in EPR is much lower than that used in NMR, so that a sample aligned beforehand in a strong field can actually be rotated during the experiment and spectra at various angles (here parallel and perpendicular to the field) recorded. To analyse the EPR spectra, a fixed-tilt (FT) model and a distributed-tilt (DT) model have been developed here to calculate the spectra expected in the twist-bend phase. Experimental spectra of samples in which the twist-bend axis should be parallel to the magnetic field showed marked differences from the spectra calculated according to the FT model but were, instead, well reproduced by the DT model. 




Fig. 11 EPR spectrum (black line) at $371.7 \mathrm{~K}$ in the supercooled $\mathrm{N}_{\mathrm{tb}}$ phase, and fit (red line) in the assumption that the local $\left\langle P_{2}\right\rangle$ is described by the Haller function shown as a dashed line in Figure 9 (see text for details).

This distribution, reported by the spin probe, is expected to be a relatively accurate description of the actual local director distribution in the twist-bend phase. The macroscopic monodomain observed in the $\mathrm{N}_{\mathrm{u}}$ phase and the fact that the values of $\left\langle P_{2}\right\rangle$ recovered from the analyses are in good agreement with those observed in a recent study of $\mathrm{CB} 11 \mathrm{CB}^{48}$ indicate that the spin probe is able to report quite accurately the overall orientation of the LC molecules, as in previous studies of uniaxial nematics $33,45,54$.

On the other hand, the EPR spin probe technique can only provide insights about the actual geometry of the twist-bend nematic phase. Our present results suggest that the tilt angle of the local director in the $\mathrm{N}_{\mathrm{tb}}$ phase is not fixed but that a relatively narrow distribution of tilt angles with $\left\langle P_{2}\right\rangle_{\mathrm{d}}$ around 0.5-0.6 is present. This observation would not be compatible with a model of the phase in which the helical axis is macroscopically straight and the tilt angle fixed. However, our results do not exclude other similar organizations. In particular the possibility of the conic angle not being unique, but rather of varying with the molecular segments of the dimers has been recently put forward by Vanakaras and Photinos $^{22}$ and could provide a possible molecular origin of the tilt distribution as reported by the CSL spin probe. A discussion about a possible more complex structure of the twist-bend phase of $\mathrm{CB} 11 \mathrm{CB}$ has also been recently provided by Mandle et al. ${ }^{9}$, while yet another possibility, recently put forward, is that the axis of the helix is not fixed but undulates ${ }^{42}$. Our data cannot positively distinguish between these different models at this stage, but we hope that this work will stimulate further analysis of these very interesting phases.

\section{Acknowledgements}

We thank MIUR, EU-BIND (FP7-216025), the University of Bologna and the CINFO Computer Centre, University of Camerino for support.

\section{References}

1 V. P. Panov, M. Nagaraj, J. K. Vij, Y. P. Panarin, A. Kohlmeier, M. G. Tamba, R. A. Lewis and G. H. Mehl, Phys. Rev. Lett., 2010, 105, 167801 1-4.

2 M. Cestari, S. Diez-Berart, D. A. Dunmur, A. Ferrarini, M. R. de la Fuente, D. J. B. Jackson, D. O. Lopez, G. R. Luckhurst, M. A. Perez-Jubindo, R. M. Richardson, J. Salud, B. A. Timimi and H. Zimmermann, Phys. Rev. E, 2011, 84, 031704 1-20.

3 R. B. Meyer, in Molecular Fluids, ed. R. Balian and G. Weil, Gordon and Breach, New York, 1976, vol. XXV of Les Houches Summer School in Theoretical Physics, pp. 272-373.

4 I. Dozov, Europhys. Lett., 2001, 56, 247-253.

5 L. Beguin, J. W. Emsley, M. Lelli, A. Lesage, G. R. Luckhurst, B. A. Timimi and H. Zimmermann, J. Phys. Chem. B, 2012, 116, 7940-7951.

6 C. Greco, G. R. Luckhurst and A. Ferrarini, Phys. Chem. Chem. Phys., 2013, 15, 14961-14965.

7 C. Meyer, G. R. Luckhurst and I. Dozov, Phys. Rev. Lett., 2013, 111, 067801 1-5.

8 V. Borshch, Y. K. Kim, J. Xiang, M. Gao, A. Jakli, V. P. Panov, J. K. Vij, C. T. Imrie, M. G. Tamba, G. H. Mehl and O. D. Lavrentovich, Nat. Commun., 2013, 4, 2635 1-8.

9 R. J. Mandle, E. J. Davis, C. T. Archbold, S. J. Cowling and J. W. Goodby, J. Mater. Chem. C, 2014, 2, 556-566.

10 D. Chen, J. H. Porada, J. B. Hooper, A. Klittnick, Y. Shen, M. R. Tuchband, E. Korblova, D. Bedrov, D. M. Walba, M. A. Glaser, J. E. Maclennan and N. A. Clark, Proc. Natl. Acad. Sci. U. S. A., 2013, 110, 15931-15936.

11 M. Gao, Y.-K. Kim, C. Zhang, V. Borshch, S. Zhou, H.-S. Park, A. Jakli, O. D. Lavrentovich, M.-G. Tamba, A. Kohlmeier, G. H. Mehl, W. Weissflog, D. Studer, B. Zuber, H. Gnaegi and F. Lin, Microsc. Res. Tech., 2014, 77, 754-772.

12 C. Meyer, G. R. Luckhurst and I. Dozov, J. Mater. Chem. C, 2015, 3, 318-328.

13 M. Tamba, S. Salili, C. Zhang, A. Jakli, G. Mehl, R. Stannarius and A. Eremin, RSC Advances, 2015, 5, 11207-11211.

14 R. J. Mandle, E. J. Davis, C.-C. A. Voll, C. T. Archbold, J. W. Goodby and S. J. Cowling, Liq. Cryst., 2015, 42, 688-703.

15 R. J. Mandle, E. J. Davis, C. T. Archbold, C. C. A. Voll, J. L. Andrews, S. J. Cowling and J. W. Goodby, Chem.-Eur. J., 2015, 21, 8158-8167.

16 C. Greco, G. R. Luckhurst and A. Ferrarini, Soft Matter, 2014, 10, 9318-9323.

17 Z. Lu, P. A. Henderson, B. J. A. Paterson and C. T. Imrie, Liq. Cryst., 2014, 41, 471-483.

18 R. J. Mandle, E. J. Davis, S. A. Lobato, C. C. A. Vol, S. J. Cowling and J. W. Goodby, Phys. Chem. Chem. Phys., 2014, 16, 6907-6915.

19 D. Chen, M. Nakata, R. Shao, M. R. Tuchband, M. Shuai, U. Baumeister, W. Weissflog, D. M. Walba, M. A. Glaser, J. E. Maclennan and N. A. Clark, Phys. Rev. E, 2014, 89, 022506 $1-5$.

20 A. Hoffmann, A. G. Vanakaras, A. Kohlmeier, G. H. Mehl and D. J. Photinos, Soft Matter, 2015, 11, 850-855. 
21 E. Gorecka, M. Salamonczyk, A. Zep, D. Pociecha, C. Welch, Z. Ahmed and G. Mehl, Liq. Cryst., 2015, 42, 1-7.

22 A. Vanakaras and D. Photinos, Soft Matter, 2016, 12, 22082220.

23 G. Barbero, L. Evangelista, M. Rosseto, R. Zola and I. Lelidis, Phys. Rev. E, 2015, 92, 030501 1-5.

24 J. H. Freed, in Spin Labeling. Theory and Applications, ed. L. J. Berliner, Academic Press, New York, 1976, ch. 3, pp. 53-132.

25 C. Bacchiocchi, I. Miglioli, A. Arcioni, I. Vecchi, K. Rai, A. Fontecchio and C. Zannoni, J. Phys. Chem. B, 2009, 113, 5391-5402.

26 H. Gopee, A. Cammidge and V. Oganesyan, Angew. Chem. Int. Ed., 2013, 52, 8917-8920.

27 J. P. Jokisaari, G. R. Luckhurst, B. A. Timimi, J. Zhu and H. Zimmermann, Liq. Cryst., 2015, 42, 708-721.

28 R. Balachandran, V. P. Panov, J. K. Vij, A. Kocot, M. G. Tamba, A. Kohlmeier and G. H. Mehl, Liq. Cryst., 2013, 40, 681-688.

29 R. Balachandran, V. P. Panov, Y. P. Panarin, J. K. Vij, M. G. Tamba, G. H. Mehl and J. K. Song, J. Mater. Chem. C, 2014, 2, 8179-8184.

30 R. Balachandran, V. P. Panov, Y. P. Panarin, M. G. Tamba, G. H. Mehl, J. K. Song and J. K. Vij, Mol. Cryst. Liquid Cryst., 2015, 611, 65-70.

31 E. Meirovitch and J. H. Freed, J. Phys. Chem., 1984, 88, 49955004.

32 A. Arcioni, C. Bacchiocchi, I. Vecchi, G. Venditti and C. Zannoni, Chem. Phys. Lett., 2004, 396, 433-441.

33 I. Vecchi, A. Arcioni, C. Bacchiocchi, G. Tiberio, P. Zanirato and C. Zannoni, J. Phys. Chem. B, 2007, 111, 3355-3362.

34 S. G. Carr, S. K. Khoo, G. R. Luckhurst and C. Zannoni, Molec. Cryst. Liq. Cryst., 1976, 35, 7-13.

35 J. H. Freed, in Electron Spin Relaxation in Liquids, ed. L. T. Muus and P. W. Atkins, Plenum Press, New York, 1972, ch. 14, pp. 387-409.

36 D. J. Schneider and J. H. Freed, in Spin Labeling. Theory and Applications, ed. L. J. Berliner and J. Reuben, Plenum Press, New York, 1989, vol. 8 of Biological Magnetic Resonance, ch. 1, pp. 1-76.

37 A. Arcioni, R. Tarroni and C. Zannoni, J. Chem. Soc.-Faraday
Trans., 1993, 89, 2815-2822.

38 P. R. Bevington, Data Reduction and Error Analysis for the Physical Sciences, McGraw-Hill, New York, 1969.

39 E. G. Virga, Phys. Rev. E, 2014, 89, 052502 1-10.

40 D. Budil, S. Lee, S. Saxena and J. Freed, J. Magn. Reson. Ser. A, 1996, 120, 155-189.

41 J. Berntsen, T. O. Espelid and A. Genz, ACM Trans. Math. Softw., 1991, 17, 452-456.

42 P. K. Challa, V. Borshch, O. Parri, C. T. Imrie, S. N. Sprunt, J. T. Gleeson, O. D. Lavrentovich and A. Jakli, Phys. Rev. E, 2014, 89, 060501 1-5.

43 C. Bacchiocchi, I. Miglioli, A. Arcioni, K. Rai, A. Fontecchio and C. Zannoni, Mol. Cryst. Liquid Cryst., 2012, 558, 127139.

44 C. Bacchiocchi, G. Foschi, I. Miglioli, S. Shoarinejad, A. Arcioni and C. Zannoni, Mol. Cryst. Liquid Cryst., 2015, 614, $2-10$.

45 V. Oganesyan, E. Kuprusevicius, H. Gopee, A. Cammidge and M. Wilson, Phys. Rev. Lett., 2009, 102, 013005 1-4.

46 J. W. Emsley, P. Lesot, G. R. Luckhurst, A. Meddour and D. Merlet, Phys. Rev. E, 2013, 87, 040501 1-4.

47 C. S. P. Tripathi, P. Losada-Pérez, C. Glorieux, A. Kohlmeier, M.-G. Tamba, G. H. Mehl and J. Leys, Phys. Rev. E, 2011, 84, 041707 1-7.

48 Z. Zhang, V. P. Panov, M. Nagaraj, R. J. Mandle, J. W. Goodby, G. R. Luckhurst, J. C. Jones and H. F. Gleeson, J. Mater. Chem. C, 2015, 3, 10007-10016.

49 J. W. Emsley, M. Lelli, H. Joy, M. G. Tamba and G. H. Mehl, Phys. Chem. Chem. Phys., 2016, 18, 9419-9430.

50 I. Haller, Prog. Solid State Ch., 1975, 10, 103-118.

51 C. Meyer, G. Luckhurst and I. Dozov, J. Mat. Chem., 2015, 3, 318-328.

52 C. Zannoni, G. F. Pedulli, L. Masotti and A. Spisni, J. Mag. Res., 1981, 43, 141-153.

53 G. Skacej and C. Zannoni, Macromolecules, 2014, 47, 88248832.

54 A. Arcioni, C. Bacchiocchi, L. Grossi, A. Nicolini and C. Zannoni, J. Phys. Chem. B, 2002, 106, 9245-9251. 
Detailed EPR spectral analyses suggest a distributed rather than a fixed tilt angle in the twist-bend nematic phase.

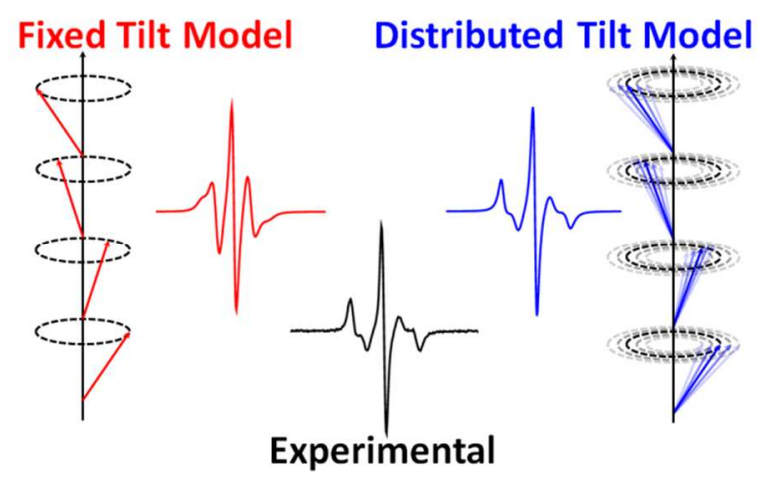

